CIENCIA Y SOCIEDAD

Volumen XVI, Número 3

Julio - Septiembre 1991

\title{
AUSENCIA DE APRENDIZAJE DE EVITACION \\ AL GUSTO SOCIALMENTE TRANSMITIDO \\ EN RATAS VIVIENDO AGRUPADAS \\ EN CONDICIONES DE LABORATORIO
}

J.R. ALBAINE PONS*

\begin{abstract}
Resumen
Ratas viviendo en pares muestran el efecto compañero envenenado, al intoxicar una de ellas con $\mathrm{LiCl}$. Este aprendizaje de evitación socialmente transmitido no ocurre si los animales viven agrupados. No aparece ni intoxicando un animal en un grupo de cinco, ni tampoco tres animales en un grupo de cinco. Se discuten los componentes sociales que interfieren con este aprendizaje.

Palabras claves: Ratas, aprendizaje, vida social
\end{abstract}

Con el descubrimiento del "Efecto García", producido con la presentación de un sabor nuevo seguido de intoxicación con $\mathrm{LiCl}$ y la subsiguiente evitación de dicho sabor en ratas, ${ }^{1.2}$ ha resultado un paradigma de inusitado interés.

Las discusiones y estudios sobre este hecho abarcan desde su utilización práctica, ${ }^{3,4}$ su importancia en la teoría del aprendizaje, ${ }^{5}$ e hipótesis sobre el significado evolutivo de la coincidencia en el tiempo de una disfunción gástrica, o sea un estado interno, con la recepción de un estímulo gustatorio, esto es con un estímulo externo. ${ }^{6.7}$ Además se han presentado trabajos sobre la acumulación de adecuación personal

* Facultad de Ciencias de la Salud, INTEC. 
(fitness) para la sobrevivencia individual, sobre la base de que ratas que coman algo que las enferme no volverán a comer de nuevo de dicha sustancia.

Este hecho, utilizando la intoxicación con $\mathrm{LiCl}$, se ha demostrado también en ratas de un día de nacidas ${ }^{8}$ y en otros mamíferos. ${ }^{9}$

Por otro lado, los hechos de que la rata es un animal altamente social ${ }^{10,11}$ y que la transmisión social de preferencias alimentarias ocurre en ratas agrupadas, ${ }^{12,13}$ parecen reforzar una serie de estudios que señalan que la conducta de evitación de alimentos puede también ser transmitida socialmente.

Experimentos han demostrado que ratas adultas que han consumido un sabor nuevo, previo a la exposición a una rata enferma que también consumió dicho sabor evitan repetir el consumo de dicha sustancia, ${ }^{14,15}$ y este fenómeno llamado "Efecto del Compañero Envenenado" o ECE, ha sido explicado como altamente adaptivo, al expresar que si un miembro de una colonia puede aprender sobre las consecuencias tóxicas de un alimento en particular, como consecuencia de la intoxicación aguda de sólo uno de sus miembros, la ventaja selectiva de tal estrategia es un omnívoro como la rata es obvia. ${ }^{16}$

El efecto ECE no ha estado sin discusión, pues varios trabajos que varían en algo la metodología original no han podido repetir el fenómeno, ni en ratas, ${ }^{17}$ ni en otros mamíferos. ${ }^{18}$

Todos los trabajos antes citados se han realizado con animales en parejas, asociando una rata enferma con otra rata sana, y hasta ahora no se han presentado trabajos con animales agrupados. Nuestro presente artículo muestra que el efecto ECE no ocurre en ratas agrupadas viviendo cinco por jaula, ni enfermando una de las cinco, ni tampoco enfermando a tres de ellas.

\section{Materiales y métodos}

Se emplearon un total de 43 ratas blancas macho obtenidas del bioterio del CESDA, en San Cristóbal, República Dominicana. El peso de los animales osciló entre 250-300 gr al realizar los experimentos. Fueron mantenidas en nuestro laboratorio bajo condiciones normales con un ciclo de LD 12:12 y agua y comida (alimento para ratas de Proteínas Nacionales, Santo Domingo, República Dominicana) por lo menos una semana antes de iniciar los procedimientos experimentales. 
Estos procedimientos se realizaron siempre durante la fase diurna del ciclo y aproximadamente a las mismas horas para cada grupo.

Procedimientos. Un primer experimento se diseñó para mostrar el efecto ECE en nuestras condiciones y en animales por pares. Se colocaron cuatro pares de ratas en 4 jaulas, donde recibían diariamente su alimento, pero el régimen de ingesta de líquidos era limitado de la siguiente manera: Los animales eran llevados a pequeñas jaulas por media hora, donde podían beber, individualmente, agua. Luego de este tiempo eran regresados a sus jaulas originales donde la pareja recibía media hora más de agua en un bebedero común para los dos animales. Se medía el consumo de agua, tanto individual, en las pequeñas jaulas, como en conjunto para el par. Este régimen se mantuvo por cinco días. En el sexto día a los animales se les ofreció en sus jaulas individuales una solución de sacarina al $1 \%$ (w/v), así como también en su bebedero común por el mismo tiempo que en los días anteriores. A una de las ratas de tres de los pares se le inyectó, inmediatamente después de ingerir la solución de sacarina en su jaula común, $10 \mathrm{ml} / \mathrm{kg}$ de peso de $0.3 \mathrm{M} \mathrm{LiCl}$ i.p. Las ratas del cuarto par se mantuvieron como controles.

El séptimo día fue el día de prueba. A cada animal se le ofreció de nuevo por media hora individual y luego media hora más en el bebedero común la solución de sacarina. La cantidad de sacarina bebida este séptimo día se tomó como índice de la magnitud de la aversión aprendida.

Un segundo experimento se diseñó para repetir el primero, pero con animales viviendo agrupados en número de cinco por jaula. Se establecieron cuatro grupos y se repitió el procedimiento anterior, inyectando al sexto día y luego de la ingesta de sacarina una rata de cada grupo en tres de los cuatro grupos, con el cuarto grupo sirviendo como control.

Un tercer experimento se diseñó con tres grupos de cinco animales por jaula, repitiendo todo el procedimiento anterior, pero inyectando, o sea enfermando, con $\mathrm{LiCl}$, tres de los cinco animales en dos de los grupos, con el tercero como control.

Los datos fueron tratados estadísticamente. ${ }^{19}$

\section{Resultados}

En nuestro primer experimento, utilizando cuatro pares de animales para probar el efecto compariero envenenado, a los seis días de la 
dieta de agua las ratas no presentaron diferencias significativas en su consumo, con un promedio de $10.5 \pm 2.20 \mathrm{ml}$ de agua $(\mathrm{P}>0.05)$, tampoco la presentaron al ingerir la solución de sacarina del sexto día $\mathrm{X}=$ $11.30 \pm 1.75 \mathrm{ml}$. Este día se inyectaron tres animales con $\mathrm{LiCl}$. Los animales inyectados quedaron acostados de lado sobre el piso de la jaula y doblados sobre sí, con los pelos erizados, señales de dolor y malestar en ratas. Al día siguiente caminaban y se comportaban a simple vista normalmente.

El séptimo día, día de la prueba, ninguno de los animales inyectados bebió la solución de sacarina, ni en su jaula individual ni en la presentación con su pareja, limitándose sólo a probarla; lo que demuestra la potencia del efecto García. Los animales apareados con los enfermos disminuyeron significativamente la ingesta de la solución de sacarina, tanto en relación con su ingestas individual del día anterior, como en comparación con la rata control. El promedio fue de un $60 \%$ menos calculado como grupo. La pareja de ratas control aumentó la ingesta de sacarina el segundo día, o día de prueba, en un 16\%. Estos resultados comprueban el ECE en ratas agrupadas por parejas. (Fig. 1)

Figura 1

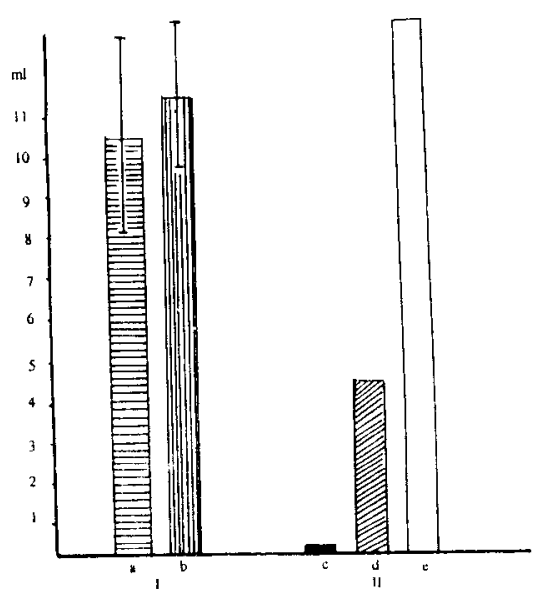

Valores promedio de ingesta de líquido en ratas viviendo en pares. I-a, ingesta de agua el último día del tratamiento, b-Ingesta de solución de sacarina por primera vez. II-Ingesta de sacarina el día de prueba: c en animales intoxicados, d en sus compañeros, e en controles. 
En nuestro segundo experimento, de cuatro grupos de cinco ratas cada uno, donde se enfermó una rata por grupo en tres de ellos, los resultados señalan que no hubo diferencia significativa en el agua ingerida por los animales el último día de privación de líquido, siendo los valores de $9.4 \pm 2.19 \mathrm{ml}, 7.2 \pm 1.09 \mathrm{ml}, 6.75 \pm 2.06 \mathrm{ml} \mathrm{y} 8.0 \pm 1.73$ $\mathrm{ml}(\mathrm{P}>0.05$, Kruskall-Wallis).

El primer día de ingestión de sacarina aparecieron diferencias significativas entre los cuatro grupos: $9.0 \pm 2.34 \mathrm{ml}, 6.8 \pm 2.48 \mathrm{ml}^{*}, 7.0$ $\pm 2.23 \mathrm{ml}^{*}$ y $11.8 \pm 2.04 \mathrm{ml}$; la ingestión de los grupos 2 y 3 fue significativamente menor al grupo $4(\mathrm{P}<0.05)$.

Una rata de los grupos 1,2 y 3 fue inyectada con $\mathrm{LiCl}$ y ninguna en el grupo 4.

En el día de prueba, el séptimo día, las ratas inyectadas casi no bebieron sacarina, dos no bebieron nada y la tercera consumió un $28 \%$ de lo que bebió el día anterior.

Las ratas miembros de los grupos que fueron expuestos a la rata intoxicada (grupos 1,2 y 3 ) bebieron el día de prueba mayor cantidad de sacarina que el día anterior y esta diferencia fue significativa (Test de signos, $\mathrm{N}=11, \mathrm{x}=2, \mathrm{P}<0.05$ ), algo que no aconteció con el grupo control, que no mostró diferencia significativa con el día anterior $(\mathrm{P}>0.05)$. Ver tabla \# 1.

Tabla I

\begin{tabular}{|c|c|c|c|}
\hline Grupo & $\begin{array}{c}\text { 1er. día Sac. } \\
(\mathrm{ml})\end{array}$ & $\begin{array}{c}\text { Día de Prueba } \\
(\mathrm{ml})\end{array}$ & Diferencia \\
\hline I $(\mathrm{n}=4)$ & $8.75 \pm 2.62$ & $10.0 \pm 2.82$ & +1.25 \\
\hline II $(\mathrm{n}=4)$ & $6.75 \pm 2.62$ & $10.25 \pm 2.87$ & +3.50 \\
\hline III $(\mathrm{n}=4)$ & $7.25 \pm 2.5$ & $12.75 \pm 2.62$ & +5.50 \\
\hline Control $(\mathrm{n}=5)$ & $11.8 \pm 2.04$ & $12.8 \pm 3.03$ & +1.0 \\
\hline
\end{tabular}

Valores promedio \pm desviación estándar de la ingestión de sacarina el primer día y el segundo (día de prueba) en ratas agrupadas que tuvieron un compañero envenenado con $\mathrm{LiCl}$ y controles que no tuvieron ningún compañero envenenado. En cada grupo hubo una rata intoxicada, que no se tomó en cuenta en estos cálculos. 
Tampoco apareció diferencia significativa entre los animales no inyectados de los grupos experimentales y el grupo control, en la cantidad de sacarina ingerida el día de prueba $(P>0.05)$.

Fue luego de encontrar que el ECE no aparecía en nuestras ratas agrupadas que pensamos que quizás una rata enferma en un grupo de cinco no sería suficiente para actuar como estímulo incondicionado, ya que en el experimento previo y en los demás citados en la literatura, donde se emplean ratas por parejas, al inyectar a una de las ratas se enferma al $50 \%$ de la población, por lơ que se diseñó el tercer experimento.

En esta prueba se intoxicaban tres ratas de un grupo de cinco luego de probar la sacarina por primera vez.

Los resultados muestran que en los dos grupos experimentales, las cuatro ratas no inyectadas bebieron el día de prueba más solución de sacarina que el primer día que la probaron, aunque esta diferencia no fue significativa estadísticamente, $\bar{X}=14.0 \pm 2.70 \mathrm{ml}$ el primer día y $\bar{X}=15.75 \pm 0.95 \mathrm{ml}$ el día de prueba. Los controles bebieron $16.0 \pm$ $1.41 \mathrm{ml} \mathrm{y} 14.4 \pm 1.67 \mathrm{ml}$ respectivamente.

\section{Discusión}

Lo primero que destaca en nuestros resultados es la reafirmación del efecto García en ratas intoxicadas. En todos nuestros experimentos la mayoría de los animales intoxicados con $\mathrm{LiCl}$ no volvió a beber de la solución de sacarina probada por vez primera minutos antes de la intoxicación, y sólo uno de los animales en todo el experimento probó de nuevo algo de líquido.

Este aprendizaje luego de un solo ensayo ha forzado a la ampliación de la base teórica del concepto de aprendizaje. Al efecto, las conocidas leyes del aprendizaje obtenidas con los modelos pavloviano y conductista presentan la repetición de ensayos como una de las condiciones para la ocurrencia de éste. ${ }^{20}$ Además, en el modelo conductista de la cámara de Skinner, es imposible enseñar a una rata a presionar una palanca para obtener comida si el alimento como refuerzo no es presentado en el rango de tres (3) segundos luego de la manipulación de la palanca y en el caso del efecto García, aún si la intoxicación ocurre siete horas después de la ingesta se presentará la evitación. ${ }^{5}$ 
Entre las varias aplicaciones que ha generado este modelo está en la actualidad la evitación en pacientes cancerosos de las aversiones al alimento que aparecen con la quimioterapia, presentando a los pacientes una bebida con un sabor distintivo previo a la medicación, para que formen la aversión a ésta y no al alimento que deben ingerir más tarde. ${ }^{4}$ Queda todavía por estudiar la magnitud de este fenómeno en animales y humanos infantes con relación a si las experiencias tempranas de este tipo son las responsables de los gustos y aversiones por cierto tipo de alimentos que experimenta el adulto.

Otro aspecto distinto es la explicación de la presencia del efecto compañero envenenado cuando hay dos ratas viviendo juntas y su no aparición en ratas viviendo en grupos de cinco.

En la situación experimental de Lavint et. al y de Bond, ${ }^{14,16}$ las parejas de las ratas a enfermar fueron introducidas en las jaulas de estas últimas donde probaron la sacarina por primera vez, y luego en sus propias jaulas fue que la probaron por segunda vez (día de prueba). Hay que notar que esta fue la primera vez que probaron la sacarina en sus propias jaulas, y por ende, parte del efecto de evitación pudo producirse por neofobia, mecanismo muy distintivo y fuerte en ratas. ${ }^{21}$ En efecto, Ellis et al. ${ }^{22}$ demostraron que en coyotes aparece evitación al alimento envenenado con el cual han tenido experiencia si éste está en un lugar nuevo para el animal, pero que es consumido si aparece en un lugar que por experiencia consideran seguro. Además se ha demostrado que la intoxicación por $\mathrm{LiCl}$ permite a un animal procesar información no sólo acerca del alimento, sino también acerca del contexto de la situación. ${ }^{23}$

Nuestro segundo experimento evita la introducción del factor neofobia puesto que ya las ratas estaban acostumbradas a beber agua en el lugar de la prueba, y más aún, se les presentó la sacarina ambas veces en este mismo lugar, por lo que se demuestra que el efecto ECE es real y poderoso en estos animales.

¿Por qué entonces no aparece en ratas agrupadas? La no aparición del efecto ECE en nuestro estudio nos obliga a evitar el modelo explicativo de las posibles ventajas que este efecto generaría en ratas viviendo en su medio natural y a buscar explicaciones alternativas.

En otra situación social se reporta un resultado que podría considerarse análogo. Gemberlin ${ }^{8}$ no encontró mediación social en el aprendizaje aversivo a la intoxicación con $\mathrm{LiCl}$ de ratas recién nacidas a ratas 
machos o a hembras nulíparas, aunque sí lo encontró con las madres. Estas últimas evitaban una solución con un sabor nuevo ingerida previo a la intoxicación de sus pequeños que todavía se amamantaban, y también se presentó el fenómeno en hembras multíparas que en ese momento no amamantaban.

Los resultados arriba expuestos nos señalan que en condiciones de vida social otros factores pueden oscurecer o debilitar el efecto ECE. De hecho se reportan estudios sobre los efectos de la densidad espacial sobre la conducta cooperativa tanto en animales como en personas y recientemente este problema ha venido enfocándose desde ángulos tanto teóricos como experimentales. ${ }^{24,25}$

Se conoce que los roedores en cuanto a dietas y estrategias alimentarias no parecen seguir las reglas de optimización ${ }^{26}$ y además que en las sociedades de ratas, al igual que en las de muchos otros animales, existe una organización jerárquica que controla y define el acceso a múltiples recursos.

Bajo esta óptica, el logro de una elevación en la escala jerárquica, al quedar temporalmente inhibidos los compañeros intoxicados, parece sobreponerse al efecto ECE. De ocurrir esto, todavía en el repertorio conductual de las ratas permanecen muchos otros mecanismos individuales que le permitirían sobrevivir, exitosamente, como lo son el propio efecto García y otros como las marcas de depósitos de excreción y orina que permiten al animal escoger un alimento a otro, ${ }^{27}$ así como utilizar "centros de información" en lugares de alimentación comunitarios. ${ }^{13,28}$

Estos mecanismos darían al individuo de un grupo mayor seguridad de supervivencia si se une, además, a un mejor y más elevado lugar jerárquico dentro de su propio grupo.

Por otro lado, información sobre estudios neurofisiológicos de los procesos aquí estudiados, señalan que el efecto García depende del área de la corteza piriforme que rodea el núcleo amigdalar del sistema límbico ${ }^{29}$ y quizás de la propia amígdala. ${ }^{30}$ Además, en ratas anósmicas no hay efecto ECE y es la amigdala límbica uno de los principales centros olfatorios del cerebro, en especial al unirse el olfato con procesos vegetativos internos y con memoria. ${ }^{31}$ Por otro lado la amígdala también participa en la formación y mantenimiento del grupo y de las jerarquías. ${ }^{32} \mathrm{La}$ conjunción de dos aspectos conductuales en un mismo 
centro cerebral nos parece un buen indicador de que ambas conductas puedan ser competitivas dados ciertos cambios ambientales. Si bien es cierto que en la naturaleza y en la actualidad, las ratas ni conviven en pares ni en pequeños grupos de cinco del mismo sexo, como en nuestro experimento, nuestros datos pudieran señalar apoyo al enfoque que dice que la selección natural se da casi siempre en favor del individuo y no del grupo ${ }^{33}$ al mostrar que la conducta individual del animal que eleva su condición en un grupo se manifiesta claramente superior a la opción de responder a señales que si bien aparecen dentro del grupo no le afectan directamente.

\section{LITERATURA CITADA}

1. García y Koelling. Relation of Cue to Consequence in Avoidance learning. Psichonomic Science. 1966, 14: 124-124.

2. García, J., R. García y Robertson. Evolution of learning mechanisms. En: Psychology of Learning. B. L. Hammonds (ed.). Washington, Amer. Psych. Ass. 1984: 191-243.

3. Harpaz, S. y J. E. Steiner. Quantitative Analysis of Feeding behavior stereotypes and of the Rejection of aversive-tasting food in the Freshwater Prawn. Macrobahiun rosenbergii. En: Olfaction and taste IX. (S.D. Roper \& J. Atema, ed.) Ann. N.Y. Academy of Sci.. Vol. 510, $1987: 347$.

4. Mattes, R.D., C. Arnold y M. Borans. Blocking Learned Food Aversions in Cancer Patients Receiving Chemotherapy, In : Olfaction and Taste IX, (S.D. Roper \& J. Atema, ed.), Ann N. Y. Academy of Sci, Vol. 510, $1987: 478$.

5. Alcock, J. Animal Behavior : An Evolutionary Approach. 2nd. Ed. Sinaver Pul. , Mass. 1979.

6. Revusky, S. The Concurrent Interference approach to Delay Learning. En : L. M. Barker, M. R. Best y M. Domjan (Eds.) Learning Mechanism of Food Selection. Waco, Tx : Baylor Univ. Press, 1977.

7. Langley W.M. y K. Knapp. Effects of Toxicosis on the predatory behavior of the golden hamster (Mesocricetus auratus) J. Comp. Psych. 1984,98 : 302-310.

8. Gemberling, G.A. Ingestion of a Novel Flavor Before Exposure to Pups Injected With Lithium Chloride Produces a Taste Aversion in Mother Rats (Rattus norvegicus). J. Comp. Psychol. $1984: 98: 285-301$.

9. Gustavson, C.R. Comparative and Field aspects of learned food aversion. En : L. M. Barger, M. R. Best y M. Domjan (Eds.) Learning Mechanisms in Food Selection. Waco, Text: Baylor Univ. Press., 1977. 
10. Wills, G. D., A.L. Wesley, F. R. Moore y D. A. Sisemore. Social interactions among rodent Conspecifics: $A$ review of experimental paradigms. Neurosci \& Behav. Rev. 1983, $7:$ 315-323.

11. Lore, R. y K. Flanelly. Rat Societies. Scientific American, May 1977: 106-116.

12. Strupp, B. J. y D. A. Levitsky. Social Transmission of food preferences in adult hooded rats (Ratus Norvegicus). Jour. Comp. Psych. 1984, 98 : 257-266.

13. Galef, B. G., Jr. Information Centers of Noway rats : Sites for information exchange and information parasitism. Animal Behav., Vol. 41 (2), 1991 : 295-302.

14. Lavin, M. J., B. Freise y S. Coombes. Transfered flavor aversions in adult rats. Behav. and Neural Biol. 1980, 28 (1): 15-33.

15. Coombes, S. ; S. H. Revusky y B. T. Lett. Long-delay taste aversion learning in a umpoisoned rat: Exposure to a poisoned rat as the unconditioned stimulus. Learning and Motivation, 1980, $11: 256-266$.

16. Bond, N. W. The poisoned-partner effect in rats: Some parametric considerations. Animal Learning and Behav., 198412 : 89-96.

17. Galef, Jr., B. ; S. W. Wigmore \& D. J. Kennett. A failure to find socially mediated taste aversion learning in norway rats (K. norvegicus). J. Comp. Psych. 1983, 97: 358-363.

18. Revusky, S.; S. Coombes \& R. Pohl. Failure of albino guinea pigs to exhibit Lavin's poisoned partner effect. Behav, and Neural Biol. 1981, $32: 111-113$.

19. Siegel, S. Estadística no paramétrica. Ed. Trillas, México, 1970.

20. Karen, Robert L. An introduction to behavior theory and its applications. Harper \& Row, New York, 1974.

21. Russell, P. A. Relationship between exploratory behavior and fear : a Review. British Jour. Psychol. 1973, $64:$ 417-433.

22. Ellis, S. R., L. Thompson \& W. E. Swanson. Effects of novelty and familiarity on illness-induced aversions to food and place cues in coyotes (Canis latrans). J. Comp. Psychol. 1983, $97:$ 302-309.

23. Kurtz, E. M. \& D. A. Levitsky. Lithitum chloride and avoidance of novel places. Behav. Newroscience, 1983, $97:$ 445-451.

24. Von Holst, D. El "stress" social en los mamiferos. Los congéneres como medio ambiente nocivo. Universitas, 1977, XV: 143-151.

25. Wesenfeld, E. La alta densidad: Su impacto y modelos para su estudio. Boletín de AVEPSO. 1990, Vol. 8: 23-33.

26. Wickery, W. L. Optimal diet models and Rodent food consumption. Animal Behav. $1984,32: 340-348$.

27. Laland, K. N. \& H. C. Plotkin. Excretory deposits surrounding food sites facilitate social learning of food preferences in Norway Rats. Animal Behaviour, 1991, 41 : 997-1006.

28. Galef, B. G. Jr.; A. Mischinger, A. Sylvie. Hungry Rats' following of conspecifics to food depends on the diets eaten by potential leaders. Animal Behaviour, 1987, 35 : 1234-1239. 
29. Dunn, L. T. \& B. J. Everitt. Double dissociations of the effects of amydala and insular cortex lesions on condicionted taste aversion, passive avoidance, and neophobia in the Rat using the excitoxin ibotenic acid. Behav. Neuroscience, $1988102: 3-23$.

30. Aggleton, J. P., M. Petrides \& S. D. Iversen. Differential effects of amydaloid lesion on conditioned taste avresion learning by rats. Physiology and Behavior, 198127 : 397-978.

31. Mishkin, M. y T. Appenzeller. The Anatomy of Memory. En: The workings of the Brain: Developinent, Memory and Perception. R. Llinás (ed.). Readings of Scientific American, W. H. Freeman and Company, N.Y. $1990: 8-104$.

32. Kling, A. Citado por Hans y Michael Eysenck. La Máquina de la Mente. Anaya, Madrid. 1982, p. 113.

33. Ruse, M. Sociobiología. Cátedra, Madrid, 1983. 